\title{
LISTA DE EVALUADORES
}

\begin{abstract}
La Revista de Española de Salud Pública cuenta en su proceso editorial con la colaboración de personas expertas en los distintos objetos de estudio y metodologías, quienes de forma anónima e independiente, actúan como evaluadores externos de los trabajos que se reciben para su publicación. Su contribución desinteresada en el proceso editorial ha sido fundamental para mantener la calidad científica alcanzada por la Revista Española de Salud Pública.
\end{abstract}

Por ello, les agradecemos especialmente su colaboración en la revisión de los trabajos efectuada a lo largo del año 2002.

CARMELO AGUIRRE GOMEZ

ALMUDENA ALAMEDA CUESTA

ÁNGEL ALBERQUILLA MENÉNDEZ-ASENJO

FRANCISCO JAVIER ALVAREZ GUTIERREZ

CARMEN AMELA HERAS

MANUEL AMEZCUA

FERNANDO ANTOÑANZAS VILLAR

JOSE MANUEL ARANDA REGULES

FERRAN BALLESTER DÍEZ

JOSE RAMÓN BANEGAS BANEGAS

GREGORIO BARRIO DE ANTA

MIQUEL A BELMONTE SERRANO

RAQUEL BOIX MARTINEZ

ANGELA BOLEA LAGUARTA

XAVIER BONFILL CUSP

ALBERT BOSCH

AURORA BUENO CAVANILLAS

CARLOS CALDERÓN

JAVIER CALLEJO GALLEGO

ROSA CANO PORTERO

LORETO CARMONA

ISABEL CASANOVAS

JOAN CARLES CASAS BAROY

OLIVIA CASTILLO SORIA

JOAN A CAYLÀ BAQUERAS

FERNANDO CHAVES SÁNCHEZ

JAVIER COLOMINA RODRÍGUEZ

FERNANDO CONDE GUTIÉRREZ

MARIA CRISTINA CUERDA COMPES

JAVIER DAMIÁN MORENO

PILAR DELGADO HITO

EMILIO DELGADO LÓPEZ-CÓZAR

MIGUEL DELGADO RODRÍGUEZ

MERCEDES DÍEZ RUIZ-NAVARRO

ELIA DÍEZ

JUAN DONADO CAMPOS

JUAN CARLOS DURO MARTÍNEZ

VICENTA ESCRIBÀ AGÜIR

J ESPEJO ESPEJO

MARIA JOSÉ FERNÁNDEZ DE SANMAMED

RAFAEL FERNÁNDEZ-CREHUET NAVAJAS

GLORIA FERNÁNDEZ-MAYORALAS

CARMEN FUENTELSAZ GALLEGO
FRANCESC GALLARDO MONTILLA

CARMEN GARCÍA COLMENERO

JULIA GARCÍA CONSUEGRA

PATRICIA GARCÍA DE OLALLA

ANA MARIA GARCÍA GARCÍA

LUIS GARCÍA OLMOS

JUAN GERVAS CAMACHO

EUGENIA GIL GARCÍA

MARIAN GIL NEBOT

JOSE MANUEL GÓMEZ SÁEZ

JESUS GONZÁLEZ ENRÍQUEZ

M. áNGELES GONZÁLEZ HEVIA

M. a ANGELES GONZÁLEZ VALENTÍN

JUAN LUIS GUTIÉRREZ FISAC

CONSUELO HUERTAS

J DE INOCENCIO

ANTONIO IÑESTA GARCIA

LUPICINIO ÍNIIGUEZ

JOSEP MARIA JANSÀ

M. ${ }^{a}$ TERESA JIMÉNEZ BUÑUALES

JAVIER LLORCA DIAZ

MARIA LUISA LÓPEZ GONZÁLEZ

CONSUELO LOPEZ NOMDEDEU

JOSE ANDRÉS LÓPEZ-HERCE CID

ELENA DE LORENZO

EMILIO LUCIO-VILLEGAS RAMOS

ANTONIO LUNA SÁNCHEZ

JOAN CARLES MARCH CERDÁ

TERESA MARTÍNEZ DEL OLMO

FERRÁN MARTINEZ NAVARRO

INMACULADA MATEO RODRÍGUEZ

BEGOÑA MERINO MERINO

ME MAESTRO CASTELBLANQUE

SANTIAGO MOLA

JUAN JOSÉ MOLINA SANZ

ARACELI MONZÓN FERNÁNDEZ

PILAR NÁJERA MORRONDO

MANEL NEBOT ADELL

FELIX OMEÑANCA TERES

VICENTE ORTÚN RUBIO

ÁNGEL OTERO PUIME

ISABEL PACHÓN

ANSELMO PEINADO 
SALVADOR PEIRÓ

JJ POLEY

MARGARITA PLA

MARINA POLLÁN SANTAMARÍA

FRANCISCO PONCE GORDO

MIQUEL PORTA SERRA

MANUEL POSADA DE LA PAZ

CONCHA PRIETO YERRO

M. ${ }^{\mathrm{a} A N G E L E S ~ P R I E T O ~}$

LUIS RAJMIL

MILAGROS RAMASCO GUTIÉRREZ

ROSA RAMÍREZ

J REDONDO CALDERON

ENRIQUE REGIDOR POYATOS

JOSE RAMÓN REPULLO LABRADOR

CARMEN DEL RIO GRACIA

JUANA ROBLEDO MARTÍN

JOSEP ROCA ANTONIO

$M^{a}{ }^{a}$ ÁNGELES RODRÍGUEZ ARENAS

FERNANDO RODRÍGUEZ ARTALEJO

JESUS RODRÍGUEZ SÁNCHEZ
ELENA RODRÍGUEZ VALÍN

MONTSERRAT ROMERA

CARLOS RUIZ FRUTOS

MANUEL JESÚS SALAS IGLESIAS

MODESTA SALAZAR AGULLÓ

TERESA SALVADOR LLIVINA

ALMUDENA SANCHEZ VILLEGAS

ANTONIO SARRIÁ SANTAMERA

ANDREU SEGURA I BENEDICTO

LUIS SEOANE PASCUAL

GUADALUPE SERRATE SANMIGUEL

MARIA JOSÉ SIERRA MOROS

CARMEN SILVESTRE BUSTO

MÓNICA SUÁREZ CARDONA

MARIA JOSÉ TORMO DIAZ

M. JOSÉ TORRES SÁNCHEZ

JOSE LUIS VÁZQUEZ BARQUERO

FERNANDO VILLAR ALVAREZ

MARTA ZIMMERMAN

MARIA VICTORIA ZUNZUNEGUI PASTOR 